Cite this: RSC Adv., 2017, 7, 51847

Accepted 1st November 2017

DOI: $10.1039 / \mathrm{c} 7 \mathrm{ra0} 8949 \mathrm{j}$

rsc.li/rsc-advances
Check for updates

Received 13th August 2017

\section{Low-voltage-driven and highly-diffractive holographic polymer dispersed liquid crystals with spherical morphology $\dagger$}

\author{
Haiyan Peng, ${ }^{a}$ Lei Yu, ${ }^{a}$ Guannan Chen, ${ }^{a}$ Trent William Bohl, ${ }^{a}$ Yunsheng Ye, ${ }^{\circ}{ }^{a}$ \\ Xingping Zhou, *ab Zhigang Xue, (D) ${ }^{a}$ V. A. L. Roy ${ }^{c}$ and Xiaolin Xie (iD *ad
}

\begin{abstract}
It is a constant pursuit to form highly-diffractive and low-voltage-driven holographic polymer dispersed liquid crystals (HPDLCs) for meeting the requirements of practical applications. Nevertheless, the highvoltage-driven characteristic is usually given while improving the diffraction efficiency of HPDLCs, and it remains a challenge to form HPDLCs with concurrent features of high diffraction and low driving voltage via a simple method. In this work, we synthesize a non-room-temperature LC, 4-butyloxy-4'cyanobiphenyl (4OCB), and mix it with a room-temperature nematic LC mixture named P0616A. These new LC mixtures are then homogeneously mixed with monomers and a photoinitibitor composed of 3,3'-carbonylbis(7-diethylaminocoumarin) (KCD) and N-phenylglycine (NPG), followed by patterning via laser interference, generating well-structured HPDLCs. The introduction of $4 O C B$ into the standard formulation is found to be able to optimize the morphology and electro-optical properties of the resulting HPDLC transmission gratings. By doping $5 \mathrm{wt} \%$ of $4 O C B$ into the HPDLCs, a high diffraction efficiency of $92 \pm 3 \%$ is obtained; meanwhile, the threshold and saturated voltages significantly decrease by $80.8 \%$ (i.e., from $12.0 \pm 0.8$ to $2.3 \pm 0.9 \vee \mu \mathrm{m}^{-1}$ ) and $73.2 \%$ (i.e., from $19.0 \pm 0.6$ to $5.1 \pm 0.7 \vee \mu \mathrm{m}^{-1}$ ), respectively, in comparison with the pristine. The enhanced performance is believed to be ascribed to the formed larger LC droplets $(70 \pm 20 \mathrm{~nm})$ and lower interface anchoring strength $\left(0.7 \mu \mathrm{N} \mathrm{m}^{-1}\right)$ of the polymer network on LCs.
\end{abstract}

\section{Introduction}

Holographic polymer dispersed liquid crystals (HPDLCs), in the form of sub-micron alternating planes of an LC-rich phase and a solid polymer-rich phase, ${ }^{1-3}$ have the easy-processing capabilities of polymers, unique electro-optical properties of LCs and controllable optical diffraction. ${ }^{4}$ Thus, HPDLCs show great application prospects in widespread advanced technique fields, such as switchable photonic crystals, ${ }^{5,6}$ 3D displays, ${ }^{7}$ distributed feedback lasers, ${ }^{8-11}$ colored 3D image storage, ${ }^{12-14}$ and radiationhardened memory for space communication..$^{15}$ Nevertheless, HPDLCs usually suffer from relatively low diffraction efficiency

${ }^{a}$ Key Laboratory for Material Chemistry of Energy Conversion and Storage, Ministry of Education, School of Chemistry and Chemical Engineering, Huazhong University of Science and Technology, Wuhan 430074, China. E-mail: xpzhou@mail.hust.edu.cn; xlxie@mail.hust.edu.cn

${ }^{b}$ Hubei Key Laboratory of Material Chemistry and Service Failure, School of Chemistry and Chemical Engineering, Huazhong University of Science and Technology, Wuhan 430074, China

'Department of Physics and Materials Science, City University of Hong Kong, Tat Chee Avenue, Kowloon Tong, Hong Kong SAR, China

${ }^{d}$ National Anticounterfeit Engineering Research Center, Wuhan 430074, China

$\dagger$ Electronic supplementary information (ESI) available. See DOI: $10.1039 / \mathrm{c} 7 \mathrm{ra} 08949 \mathrm{j}$ or high switching voltage, ${ }^{13,14,16}$ which hinders their practical applications.

To optimize the diffraction efficiency and driving voltage of HPDLCs, it is necessary to comprehend the formation mechanism of HPDLC gratings in theory. When two coherent laser beams irradiate the homogeneous mixture consisting of reactive monomers, non-reactive LCs, photosensitizer and coinitiator, the photosensitizer absorbs photons and then transforms to its excited states, which subsequently reacts with the co-initiator through photoinduced electron transfer and proton transfer. This photo-triggered reaction generates radicals to preferentially initiate reactive monomers to polymerize in the bright (interference strengthening) regions. The consumption of monomers in the high light intensity regions results in diffusion of reactive monomers from the low light intensity regions to the high light intensity regions, thereby causing a periodically spatial modulation of the polymerization kinetics. As the miscibility gap between LCs and growing polymer host increases, the radical-inert LC molecules are squeezed into the low light intensity regions in order to balance the chemical potential. Eventually, the dark (interference weakening) regions become enriched with LC molecules while the bright regions are filled with polymer, in the form of spatially alternating layers of polymer-rich and LCs-rich phases. Only the diffusion of LCs 
is faster than their nucleation and the nucleation is faster than the polymerization gelation, can a well-defined grating be formed, affording high diffraction efficiency. Through careful selection of LC concentration, ${ }^{\mathbf{1 7}, \mathbf{1 8}}$ monomer type and average functionality, ${ }^{16,18-25}$ sensitizer and co-initiator, ${ }^{13,14,20,26}$ or judicious optimization of the processing conditions such as recording temperature, light intensity and exposure time, ${ }^{17}$ the micromorphology of HPDLCs can be well controlled to offer high diffraction efficiency. Nevertheless, the electro-optical properties of HPDLCs are also closely dependent on their micromorphology, and HPDLC gratings with high diffraction efficiency usually need a high driving voltage to switch. ${ }^{12,13,16,23}$ Therefore, it remains a great challenge to chase low driving voltage while maintaining a high diffraction efficiency of HPDLCs.

To overcome this bottleneck, two typical methods have been employed so far. The first method is to decrease the interface anchoring energy between the polymer host and LC by reducing the interfacial tension with the introduction of surfactants or low surface energy polymers containing fluorine. ${ }^{27-32}$ For instance, $\mathrm{Xu}$ et al. investigated the effect of surfactants on the electro-optical properties of HPDLCs by adding a small portion of three different kinds of surfactants, namely, octanoic acid, sorbitan monooleate, and Tergital Min-Foam 1X. ${ }^{32}$ Although the threshold electric field was reduced from $13.0 \mathrm{~V} \mu^{-1}$ to about $2.3 \mathrm{~V} \mu \mathrm{m}^{-1}$ by adding $8 \mathrm{wt} \%$ of sorbitan monooleate, the diffraction efficiency was not higher than $70 \%$. Bunning's group discovered that, to some extent, adding octanoic acid reduced the switching voltages of HPDLCs. ${ }^{31}$ However, octanoic acid contaminated the LC droplets, which reduced the clearing temperature and potentially gave rise to long-term performance degradation issues. ${ }^{31}$ Woo and Kim confirmed that the switching voltages of HPDLCs could be significantly decreased by incorporating the partially fluorinated acrylate monomers because of the decrease in the interface anchoring strength. ${ }^{27}$ However, fluoride monomers are usually expensive and hard to synthesize. The second method to lower down driving voltage is to incorporate inorganic nanoparticles with relatively large lowfrequency electrical conductivity, such as silica, ${ }^{3,34}$ gold or silver, ${ }^{35,36}$ carbon nanotubes, ${ }^{37,38}$ graphene or graphene oxide, ${ }^{39,40}$ and $\mathrm{ZnS}^{16}$ into the polymer-rich regions of HPDLC gratings. Fontecchio's group experimentally proved a reduction in switching voltage of HPDLCs by doping a low concentration of oxidized multi-walled carbon nanotubes, due to the fact that the MWNTs got trapped in the polymer matrix to improve the matrix conductivity. ${ }^{38}$ Braun and co-workers incorporated acrylate-functionalized silica nanoparticles into HPDLC gratings and demonstrated that the functionalized silica nanoparticles were predominantly sequestered in the polymer-rich regions, which afforded lower driving voltage and a relatively high diffraction efficiency. ${ }^{34}$ Recently, our group revealed that the threshold driving voltage of HPDLCs could be decreased


to $94 \pm 2 \%$ when doping $8 \mathrm{wt} \%$ of $\mathrm{ZnS}$ semiconductor nanoparticles into the polymer-rich regions. ${ }^{16}$ The drawback of nanoparticle doping is that complicated synthesis and surface functionalization are usually involved. Despite of the extensive research and amazing progress, it remains a big challenge to chase the low driving voltage while maintaining a high diffraction efficiency of HPDLCs via a simple way.

Herein, a novel approach is proposed which relies on simple LC mixing. LC mixing is a long-standing method to achieve desired LC properties for meeting the requirement of display. We hypothesize that this simple approach is able to offer a fine tune in the micromorphology of HPDLCs which is envisioned to consequently offer enhanced device performance. We first synthesized the LC 4OCB easily from low cost raw materials via a one-step process, and then mixed it with a commercially available nematic LC mixture named P0616A. These new LCs were then homogeneously mixed with monomers and the photoinitibitor composed of KCD and NPG. HPDLCs were finally generated by exposing the mixtures to laser interference patterns. The LCs 4OCB and P0616A jointly form the LCs-rich regions during the formation of HPDLCs due to their better compatibility compared with the poor compatibility with polymer. The LC 4OCB can not only control the micromorphology as manifested by the significant increased average diameter of LC droplets, but it can also decrease the interface anchoring energy of the polymer on LCs. HPDLC transmission gratings with a high diffraction efficiency of $92.0 \pm 2.8 \%$, a low threshold driving voltage of $2.3 \mathrm{~V} \mathrm{\mu m}^{-1}$, and a low saturated voltage of $5.1 \mathrm{~V} \mu \mathrm{m}^{-1}$ were achieved by doping $5 \mathrm{wt} \%$ of 4 OCB into the HPDLCs. The new findings here are believed to advance the understanding of the structure-property relation of HPDLCs, and the proposed protocol here promises practical application of HPDLCs with concurrent high diffraction efficiency and low driving voltage.

\section{Materials and methods}

\subsection{Materials}

4'-Hydroxy-4-biphenylcarbonitrile (purity 99\%), 1-butyl bromide (purity 99\%), and sodium hydroxide $(\mathrm{NaOH}, \mathrm{AR})$ were purchased from Sinopharm Chemical Reagent Co., Ltd., China. $N, N$-Dimethylacrylamide (DMAA, purity 98\%) was purchased from J\&K Scientific. Hyperbranched acrylate monomer 6361-100 was donated by Eternal Chemical Co., Ltd., China. 3,3'Carbonylbis(7-diethylaminocoumarin) (KCD) and $N$-phenylglycine (NPG) were received from Aldrich and Aladdin, respectively. The nematic LC mixture named P0616A (Table 1) was obtained from Shijiazhuang Chengzhi Yonghua Display Material Co., Ltd., China.

\subsection{Synthesis of LC 4-butyloxy-4'-cyanobiphenyl (4OCB)}

The LC 4OCB was synthesized as follows according to the reported procedure: ${ }^{41} 1.95 \mathrm{~g}(10 \mathrm{mmol})$ of 4 '-hydroxy-4biphenylcarbonitrile and $0.4 \mathrm{~g}(10 \mathrm{mmol})$ of $\mathrm{NaOH}$ were placed in a three neck round bottom flask fitted with a condenser. Nitrogen gas was passed through the apparatus. $30 \mathrm{~mL}$ of anhydrous butanone was then added via a cannula, followed by vigorous stirring for $20 \mathrm{~min}$ at $60{ }^{\circ} \mathrm{C}$ using a magnetic stirring bar. Next, $10 \mathrm{~mL}(9 \mathrm{mmol})$ of 1-butyl bromide in butanone was dropwise added and the mixture was 
Table 1 Chemical components of the nematic LC mixture P0616A

\begin{tabular}{|c|c|c|c|c|}
\hline $\mathrm{LC}$ & Structural formula & Percentage/wt\% & Melting point $/{ }^{\circ} \mathrm{C}$ & Clear point $/{ }^{\circ} \mathrm{C}$ \\
\hline $5 \mathrm{CB}$ & & 56.5 & 23.0 & 35.0 \\
\hline $7 \mathrm{CB}$ & & 25.1 & 28.5 & 42.0 \\
\hline $8 \mathrm{OCB}$ & & 11.4 & 54.5 & 75.0 \\
\hline
\end{tabular}

kept refluxing for $19 \mathrm{~h}$ under the protection of nitrogen gas. The resulting solution was filtered and $100 \mathrm{~mL}$ of $\mathrm{NaOH}$ water solution $\left(0.4 \mathrm{~mol} \mathrm{~L}^{-1}\right)$ was added to the filtrate. The organic product in the filtrate was extracted using dichloromethane for three times, and then concentrated using rotary evaporation under reduced pressure. The resulting solid was purified by recrystallization from ethanol. The final product (white crystals, yield: $70 \%$ ) was characterized by nuclear magnetic resonance spectroscopy (NMR, Bruker, AV400) and high resolution mass spectrometry (HRMS, Bruker SolariX 7.0T FTMS).

\subsection{Phase transition characterization of $40 C B$ and $40 C B /$ P0616A mixtures}

The phase transition behaviors of $40 \mathrm{CB}$ and $4 \mathrm{OCB} / \mathrm{P} 0616 \mathrm{~A}$ mixtures were determined using differential scanning calorimetry (DSC, TA Q2000) and cross polarized optical microscopy (POM, Axio Scope. A1, Carl Zeiss).

The LC mixtures composed of $40 \mathrm{OCB}$ and P0616A were prepared in selected proportions of $0 / 33,2 / 31,5 / 28,8 / 25$ and $11 /$ 22 , respectively. To obtain homogeneous LC mixtures, the LCs were heated to the isotropic phase and annealed for a period of $30 \mathrm{~min}$.

\subsection{Preparation of holographic mixtures}

The holographic mixtures used in this study consisted of the mono-functional monomer DMAA, hyperbranched acrylate monomer 6361-100, a photoinitibitor composed of KCD and NPG, as well as LC P0616A and 4OCB. The weight fraction of each component is shown in Table 2. The total weight percentage of 4OCB and P0616A was kept unchanged in the monomer/LCs mixtures (33 wt $\%$ in total). Thus, the LC 4 OCB

Table 2 Chemical components for holography (by weight ${\text { percentage })^{a}}$

\begin{tabular}{lrlll}
\hline Entry & 4OCB & P0616A & DMAA & $6361-100$ \\
\hline 1 & 0.0 & 33.0 & 44.7 & 22.3 \\
2 & 2.0 & 31.0 & 44.7 & 22.3 \\
3 & 5.0 & 28.0 & 44.7 & 22.3 \\
4 & 8.0 & 25.0 & 44.7 & 22.3 \\
5 & 11.0 & 22.0 & 44.7 & 22.3
\end{tabular}

${ }^{a}$ The KCD and NPG concentrations were set as 0.6 and $1.3 \mathrm{wt} \%$ of the total mass of monomers and LCs, respectively. concentration in the monomer/LCs mixtures was calculated to increase from zero to $11 \mathrm{wt} \%$. The monomers and LCs were then added into brown glass bottles and ultrasonicated at $30{ }^{\circ} \mathrm{C}$ for $10 \mathrm{~min}$ to obtain homogeneous mixtures. Finally, KCD and NPG were introduced to the mixtures, followed by another 10 min of ultrasonication.

\subsection{Viscosity of holographic mixtures}

The viscosities of holographic mixtures were characterized at $25{ }^{\circ} \mathrm{C}$ using a rheometer (MCR 302, Anton-Paar) with two parallel plates in a diameter of $25 \mathrm{~mm}$. A shear rate of 0-100 s was employed, and the gap between the two plates was set as $0.2 \mathrm{~mm}$.

\subsection{Photopolymerization kinetics}

Photopolymerization kinetics was investigated using a Q2000 photo-differential scanning calorimeter (P-DSC, TA Instruments). About $7 \mathrm{mg}$ of the sample was added in an aluminum liquid pan that was then placed onto the sample holder while another vacuum aluminum pan was placed onto the reference holder. The sample was purged by $50 \mathrm{~mL} \mathrm{~min}^{-1}$ of nitrogen gas during the test. After being kept isothermal at $30^{\circ} \mathrm{C}$ for $5 \mathrm{~min}$, the aluminum pans were simultaneously irradiated by two separate monochromatic $442 \mathrm{~nm}$ beams from the top isothermally for another $20 \mathrm{~min}$. The light intensity for each beam was fixed at $3.2 \mathrm{~mW} \mathrm{~cm}^{-2}$. The exothermal heat flow during photomediated reaction versus the irradiation time was recorded, which allowed for the calculation of photopolymerization rate and double-bond conversion using the previously reported method, ${ }^{23}$

$$
\begin{aligned}
& R_{\mathrm{p}}=(\mathrm{d} H / \mathrm{d} t) /\left(\sum \frac{x_{\mathrm{i}} m f_{\mathrm{i}} \Delta H_{0}}{M_{\mathrm{i}}}\right) \\
& \alpha(t)=\Delta H(t) /\left(\sum \frac{x_{\mathrm{i}} m f_{\mathrm{i}} \Delta H_{0}}{M_{\mathrm{i}}}\right)
\end{aligned}
$$

where, $R_{\mathrm{p}}$ is the rate of photopolymerization at the reaction time $t$, and $\alpha(t)$ is the $\mathrm{C}=\mathrm{C}$ double bond conversion up to time $t . \mathrm{d} H /$ $\mathrm{d} t$ and $\Delta H(t)$ are the exothermic heat flow and enthalpy change, respectively. $m, x_{\mathrm{i}}, f_{\mathrm{i}}$ and $M_{\mathrm{i}}$ are the total mass of holographic mixtures, individual weight fraction, functionality and molecular weight of the monomer i, separately. $\Delta H_{0}$ represents the standard reaction enthalpy of $\mathrm{C}=\mathrm{C}$ double bond that equals $86 \mathrm{~kJ} \mathrm{~mol}^{-1}$ for acrylate and $83 \mathrm{~kJ} \mathrm{~mol}^{-1}$ for acrylamide. 


\subsection{Photorheology}

The photorheological behavior of holographic mixtures under flood light exposures was investigated using a rheometer (MCR 302 , Anton-Paar) that was equipped with two parallel plates with diameters of $25 \mathrm{~mm}$, wherein the bottom plate was transparent polyester that allowed the light to penetrate through. To obtain rational experimental results, the gap between the two plates, angular frequency, strain, and temperature were set as $0.1 \mathrm{~mm}$, $10 \mathrm{rad} \mathrm{s}^{-1}, 15 \%$ and $25^{\circ} \mathrm{C}$, respectively. Under the protection of

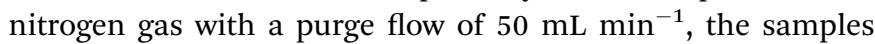
were isothermally exposed to $3.2 \mathrm{~mW} \mathrm{~cm}^{-2}$ of monochromatic $442 \mathrm{~nm}$ light for a proper duration after being sheered in dark for $1 \mathrm{~min}$. Both the storage and loss moduli were recorded and their crossover was considered the gelation point. ${ }^{\mathbf{4 2 , 4 3}}$

\subsection{Holographic recording}

To fabricate the transmission holographic gratings, the holographic mixtures were injected by capillary action into a LC cell with an $11 \mu \mathrm{m}$ thick gap (confirmed by LCT-5016C liquid crystal display parameter tester from North LC Engineering Research and Development Centre, China). A $442 \mathrm{~nm} \mathrm{He-Cd} \mathrm{laser} \mathrm{beam}$ was divided by a splitter into two separate beams with an equal intensity of $3.2 \mathrm{~mW} \mathrm{~cm} \mathrm{~cm}^{-2}$. Then, the two coherent beams were used to simultaneously irradiate the holographic mixtures sandwiched in the LC cell for $40 \mathrm{~s}$ (Scheme 1), followed by postcure for another 5 min using a UV lamp. The bisector of the external angle between the two coherent laser beams was normal to the planar surface of LC cell.

\subsection{Electro-optical performance}

The diffraction efficiencies of the HPDLCs gratings were measured by the LCT-5016C display parameter tester. During the test, a $p$-polarized $633 \mathrm{~nm}$ laser with high collimation (Thorlabs, USA) was employed to non-destructively probe the holographic gratings at the Bragg angle. The diffraction efficiency was defined as the ratio of the first-order diffraction intensity to the total intensities of the diffraction and transmission beams. For implementing the electric switching, a square wave voltage up to $250 \mathrm{~V}$ with a frequency of $1 \mathrm{kHz}$ was applied. Three to five independent samples were characterized and each result was given in the form of average value plus standard deviation.

\subsection{Morphology characterization}

The HPDLCs inside glass cells were cut into small specimens and then soaked in $n$-hexane for $48 \mathrm{~h}$ to fully remove the 4OCB and P0616A from the gratings. After being dried in air and coated with a thin platinum layer on the top surface, the morphology was characterized under a field emission scanning electron microscope (Sirion 200 FESEM). Atomic force microscopy (AFM, Shimadzu SPM-9700) was used to investigate the surface topology of HPDLCs transmission gratings after LC removal. The images were acquired in the tapping mode with a resonant frequency of $300 \mathrm{kHz}$.

\section{Results and discussion}

\subsection{Characterization of the LC $40 C B$}

The successful synthesis of 4OCB is confirmed by NMR, HRMS and elemental analysis. As shown in Fig. 1, ${ }^{1} \mathrm{H}-\mathrm{NMR}$ spectral information of the LC 4 OCB is given as follows, $(400 \mathrm{MHz}$, $\left.\mathrm{CDCl}_{3}\right) \delta[\mathrm{ppm}]: 7.59(2 \mathrm{H}$, ortho to $\mathrm{CN}), 7.53(2 \mathrm{H}$, meta to $\mathrm{CN})$, $7.43\left(2 \mathrm{H}\right.$, meta to $\left.\mathrm{OCH}_{2}-\right), 6.90\left(2 \mathrm{H}\right.$, residual ortho to $\left.\mathrm{OCH}_{2}-\right)$, $3.92\left(2 \mathrm{H}, \mathrm{PhOCH}_{2} \mathrm{CH}_{2}^{-}\right), 1.71\left(2 \mathrm{H}, \mathrm{PhOCH}_{2} \mathrm{CH}_{2}-\right), 1.43(2 \mathrm{H}$, $\left.-\mathrm{CH}_{2} \mathrm{CH}_{3}\right), 0.90\left(3 \mathrm{H},-\mathrm{CH}_{2} \mathrm{CH}_{3}\right)$. The ${ }^{13} \mathrm{C}-\mathrm{NMR}$ spectrum of $4 \mathrm{OCB}$ is displayed in Fig. $\mathrm{S} 1$ in the ESI, $\dagger$ and the spectral information is as follows, $\left(400 \mathrm{MHz}, \mathrm{CDCl}_{3}\right) \delta[\mathrm{ppm}]: 159.86,145.26,132.52$, $131.20,128.28,127.03,119.07,115.10,110.03,67.80,31.28$, 19.26, 13.85. HRMS of 4OCB is shown in Fig. S2 in the ESI, $\dagger$ calculated for $\left[\mathrm{C}_{17} \mathrm{H}_{18} \mathrm{NO}\right]^{+}\left(\mathrm{MH}^{+}\right)$: $\mathrm{m} / z$ 252.1344; found: 252.1378. Elemental analysis (by weight): N 5.50\%, C $80.43 \%, \mathrm{H}$ $6.70 \%$, O $7.37 \%$.

The phase behavior of 4 OCB was investigated using DSC and POM. As displayed in Fig. 2a, a big melting peak at $78^{\circ} \mathrm{C}$ is clear with an enthalpy value of $83.6 \mathrm{~J} \mathrm{~g}^{-1}$ during the second heating. When cooling down from $150{ }^{\circ} \mathrm{C}$, a weak exothermic peak appears at $74^{\circ} \mathrm{C}$ with an enthalpy value of $1.8 \mathrm{~J} \mathrm{~g}^{-1}$. This weak peak is ascribed to the isotropic-nematic transition, ${ }^{44}$ which is confirmed by the uniaxial Maltese cross texture in tetrapode
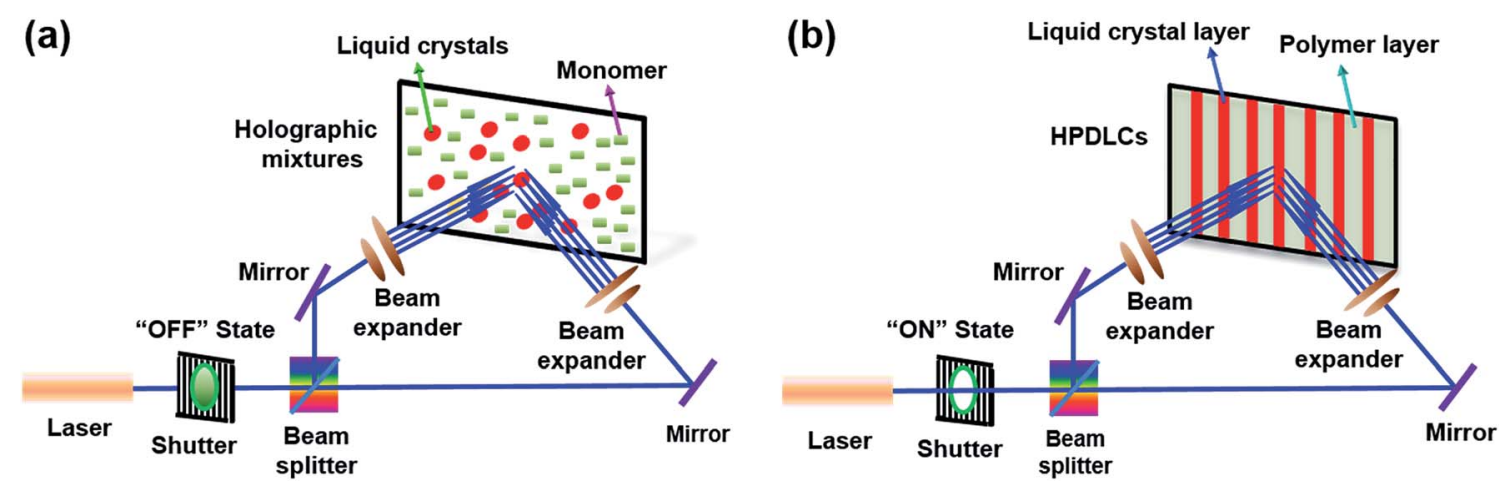

Scheme 1 Schematic illustration of the holographic recording of transmission HPDLCs where gratings are formed by opposite diffusion of liquid crystals and monomer. (a) Before holographic polymerization (the shutter is in the off state). (b) After holographic polymerization (the shutter is in the on state). 


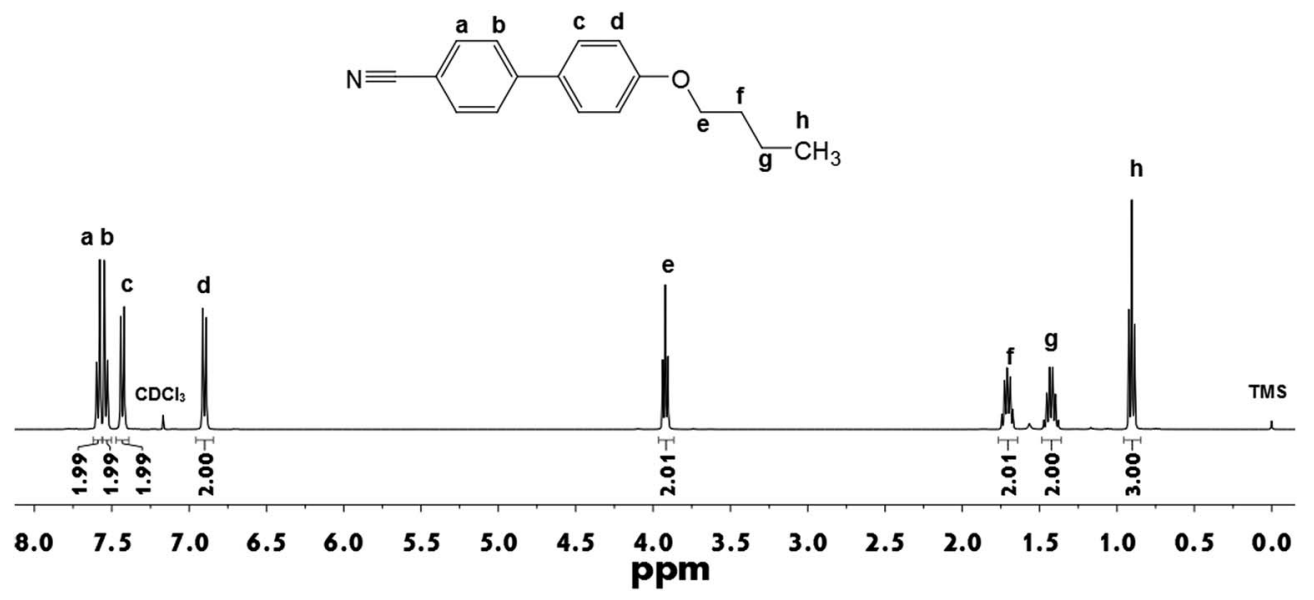

Fig. $1{ }^{1} \mathrm{H}$-NMR spectrum of the LC 4 OCB in the solvent $\mathrm{CDCl}_{3}$.

nematic droplets under POM (Fig. 2b). ${ }^{45}$ When further cooling the sample, a relatively large exothermic peak $\left(64.4 \mathrm{~J} \mathrm{~g}^{-1}\right)$ appears at $43{ }^{\circ} \mathrm{C}$ corresponding to the crystallization of 4OCB. The nematic phase was observed only when cooling the isotropic melt, which indicates that the 4OCB is a monotropic nematic LC. ${ }^{46}$ The influence of the 4 OCB on the phase transition temperature and texture of the LCs P0616A are illustrated in Fig. 2c and d, respectively. When varying the mass ratio between the LCs 4OCB and P0616A from 0/33 to $2 / 31,5 / 28,8 / 25$ and 11 / 22 , the nematic-isotropic transition temperature of the mixtures is 58, 59, 61, 62 and $63{ }^{\circ} \mathrm{C}$, respectively (Fig. 2c), indicating that the nematic phase is easier to form when increasing the $4 \mathrm{OCB}$ in the LC mixtures. Adding 4OCB does not influence the schlieren texture with dark brushes of P0616A, as shown in Fig. 2d.
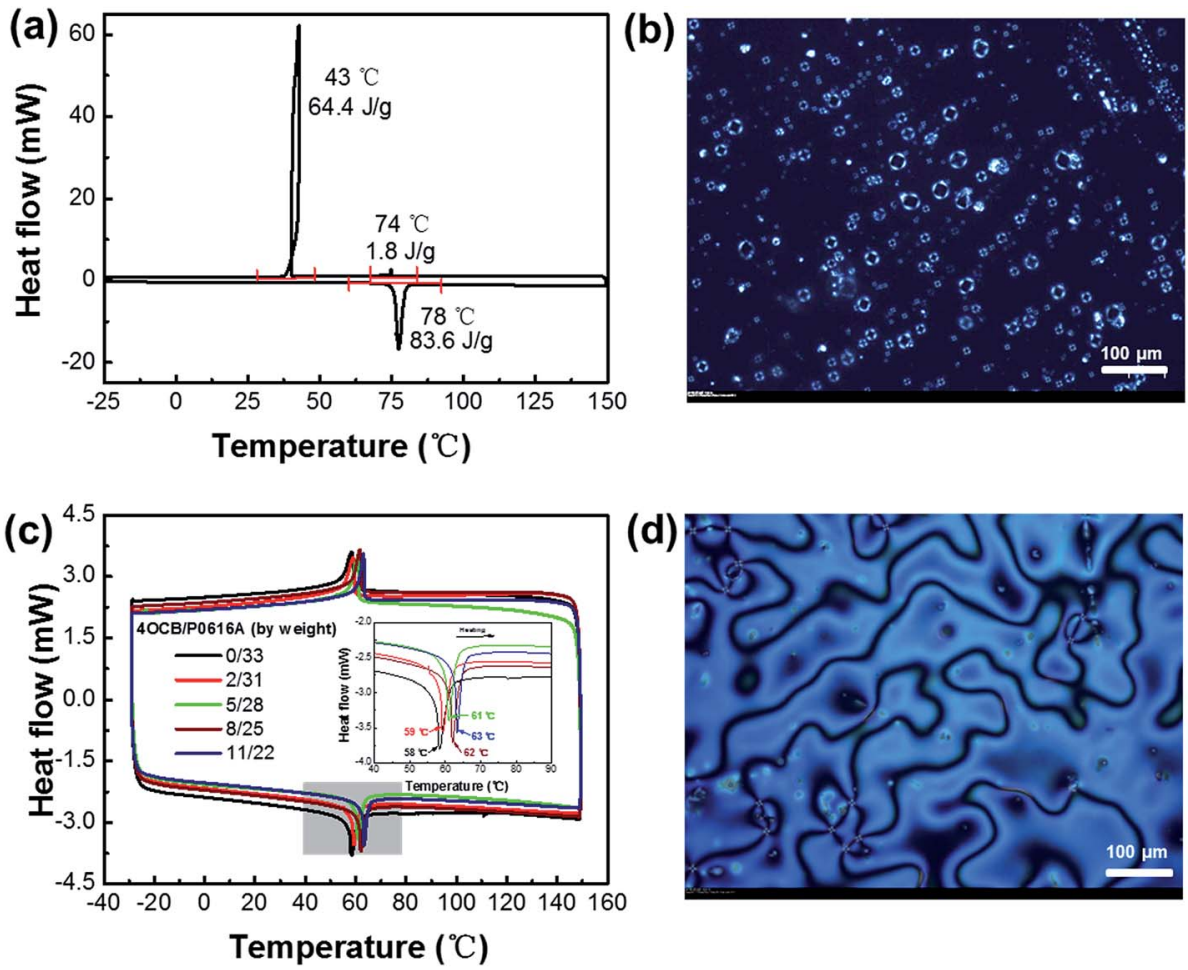

Fig. 2 (a) DSC curve of the $4 \mathrm{OCB}$ during the second heating and cooling at a rate of $5^{\circ} \mathrm{C} \mathrm{min}^{-1}$ between -25 and $150{ }^{\circ} \mathrm{C}$. (b) Maltese cross texture of the $4 \mathrm{OCB}$ tetrapode droplets at $70^{\circ} \mathrm{C}$ during the second cooling at a rate of $2{ }^{\circ} \mathrm{C} \min ^{-1}$ from 120 to $70{ }^{\circ} \mathrm{C}$, indicating the uniaxial nematic phase. (c) DSC curves of the LC mixtures with varied mass ratios of $40 C B$ and P0616A. The data were obtained during second heating and cooling at a rate of $10^{\circ} \mathrm{C} \mathrm{min}^{-1}$ between -30 and $150^{\circ} \mathrm{C}$. (d) Schlieren texture with dark brushes of the $40 \mathrm{OB} / \mathrm{P0616} \mathrm{A}$ mixture in a mass ratio of $5 / 28$ at $58{ }^{\circ} \mathrm{C}$ during the second cooling at a rate of $2{ }^{\circ} \mathrm{C} \mathrm{min}{ }^{-1}$ from 120 to $58^{\circ} \mathrm{C}$. Samples were sandwiched between an ordinary glass slide and a coverslip when conducting the POM characterization. 


\subsection{Photopolymerization kinetics and photorheology}

The photopolymerization rate as a function of double-bond conversion and the viscosity of the holographic mixtures with varied 4OCB content are displayed in Fig. 3a and b, respectively. The introduction of 4OCB shows a small influence on the photopolymerization kinetics and viscosity of holographic mixtures. With an augmentation of the 4OCB content from zero to $11 \mathrm{wt} \%$, the maximum photopolymerization rate and viscosity increased by $12.8 \%$ (i.e., from $3.9 \times 10^{-3}$ to $4.4 \times 10^{-3}$ $\mathrm{s}^{-1}$ ) and $7.4 \%$ (i.e., from 6.8 to $7.3 \mathrm{mPa} \mathrm{s}^{-1}$ ), respectively. To be noted that, it is impractical to analyze the molecular weight of the generated polymer as it is a cross-linked network. However, the almost unchanged double-bond conversion when varying the 4OCB content implies that the molecular weight of the generated polymer shows insignificant influence on the HPDLC structure and performance. The photorheological behavior during photopolymerization is displayed in Fig. 3c. Before turning on the light, it can be seen that the initial loss modulus is greater than the storage modulus; however, both the loss modulus and storage modulus increase when the light irradiation duration is extended because the molecular weight increases during photopolymerization. When further increasing the irradiation time, the storage modulus increases faster than the loss modulus does, and the time corresponding to their crossover is considered to be the gelation time. When the 4 OCB content is $0,2,5,8$ and $11 \mathrm{wt} \%$, the gelation time during photopolymerization is found to be $63 \pm 6,63 \pm 11,51 \pm$ 9, $55 \pm 10$ and $58 \pm 7$ s, respectively (Fig. 3d).

\subsection{Effect of LC 4OCB content on the micromorphology of HPDLCs gratings}

The performance of HPDLC gratings inherently depends on their micromorphologies. As illustrated in Fig. 4, dark channels or holes represent the original LCs before being removed and the others represent polymer regions. Uniform gratings with sharp edges were obtained except for the grating with $5 \mathrm{wt} \%$ of 4OCB where dark holes represent the original locations of LC droplets (Fig. 4c). The LCs-rich regions are composed of small, discrete, and nearly spherical LC droplets with an average size of $70 \pm 20 \mathrm{~nm}$, which are slightly larger than those found in the other gratings. The widths of LCs-rich regions with the incremental LC 4OCB content are approximately $0.34 \pm 0.04,0.31 \pm$ $0.02,0.36 \pm 0.05,0.27 \pm 0.05$ and $0.23 \pm 0.02 \mu \mathrm{m}$, and they are separated by polymer-rich regions with widths of about $0.77 \pm$ $0.08,0.77 \pm 0.07,0.76 \pm 0.02,0.75 \pm 0.04$ and $0.78 \pm 0.03 \mu \mathrm{m}$, respectively. We therefore know that the ratio of the LCs-rich region width to the grating period $(44 \%, 40 \%, 47 \%, 36 \%$ and $29 \%$, respectively) generally decreases with the incremental LC $4 \mathrm{OCB}$, except for the grating with $5 \mathrm{wt} \%$ of $4 \mathrm{OCB}$.

Theoretically, the phase separation of HPDLC gratings strongly depends on the relative rates of monomer/LC diffusion, polymerization gelation and LC nucleation. ${ }^{47}$ Only if the diffusion of LCs is faster than their nucleation and the nucleation is faster than the polymerization gelation can a laminated grating structure be formed, as illustrated in Fig. 4a. Because the viscosity does not change significantly when loading $4 \mathrm{OCB}$, the molecular diffusion difference can be negligible. With the
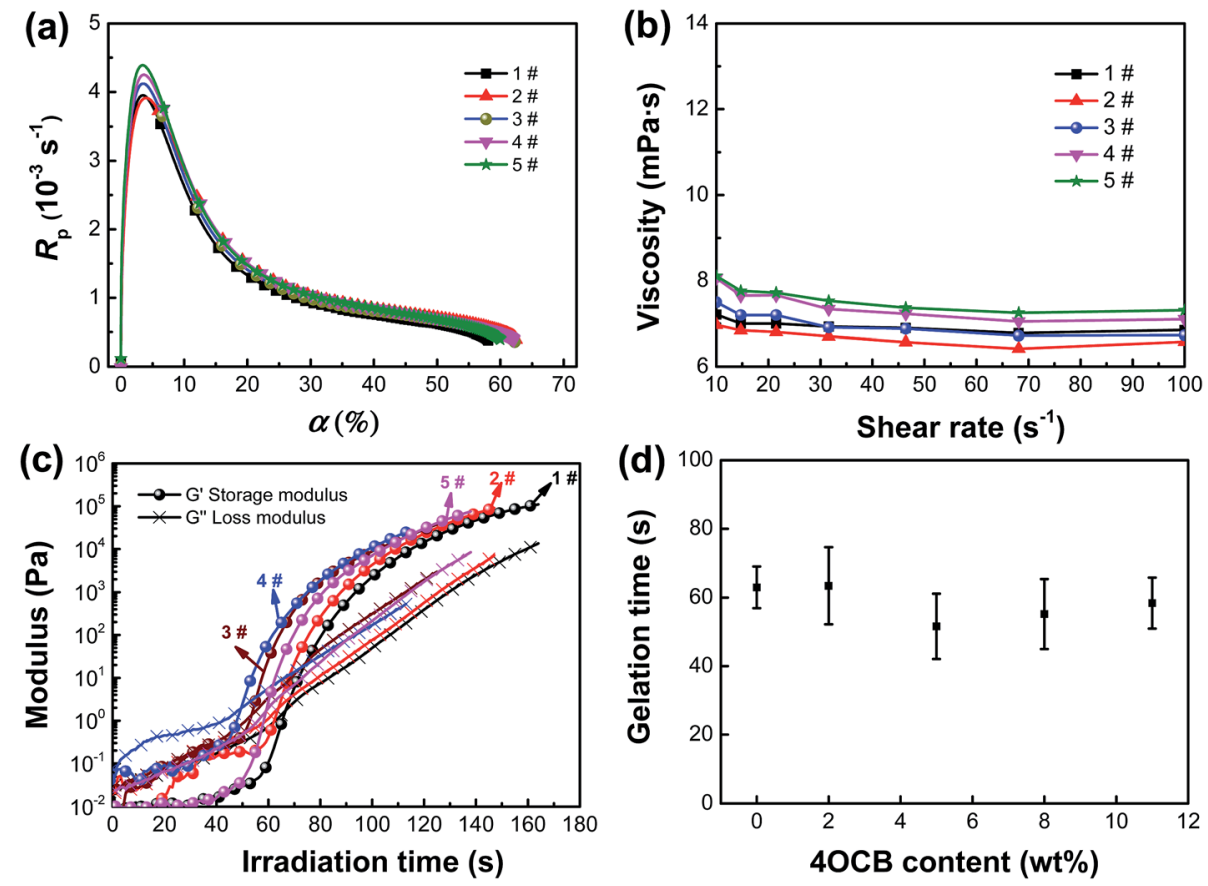

Fig. 3 (a) Polymerization rate $R_{\mathrm{p}}$ versus double bond conversion $\alpha$ for homogeneous mixtures containing varied content of $40 \mathrm{OCB}$ upon exposure to a monochromatic $442 \mathrm{~nm}$ visible light with a light intensity of $3.2 \mathrm{~mW} \mathrm{~cm}^{-2}$. (b) Viscosity of the holographic mixtures with different $40 C B$ content as a function of shear rate. (c) Storage $\left(G^{\prime}\right)$ and loss $\left(G^{\prime \prime}\right)$ moduli versus irradiation time upon exposure to $3.2 \mathrm{~mW} \mathrm{~cm}^{-2}$ of $442 \mathrm{~nm}$ light for the mixtures containing varied concentrations of LC 4OCB. (d) Gelation time versus LC $4 O C B$ content. The LC 4 OCB content is $0,2,5,8$ and $11 \mathrm{wt} \%$ for the entry $1 \#, 2 \#, 3 \#, 4 \#$ and 5\#, respectively. 

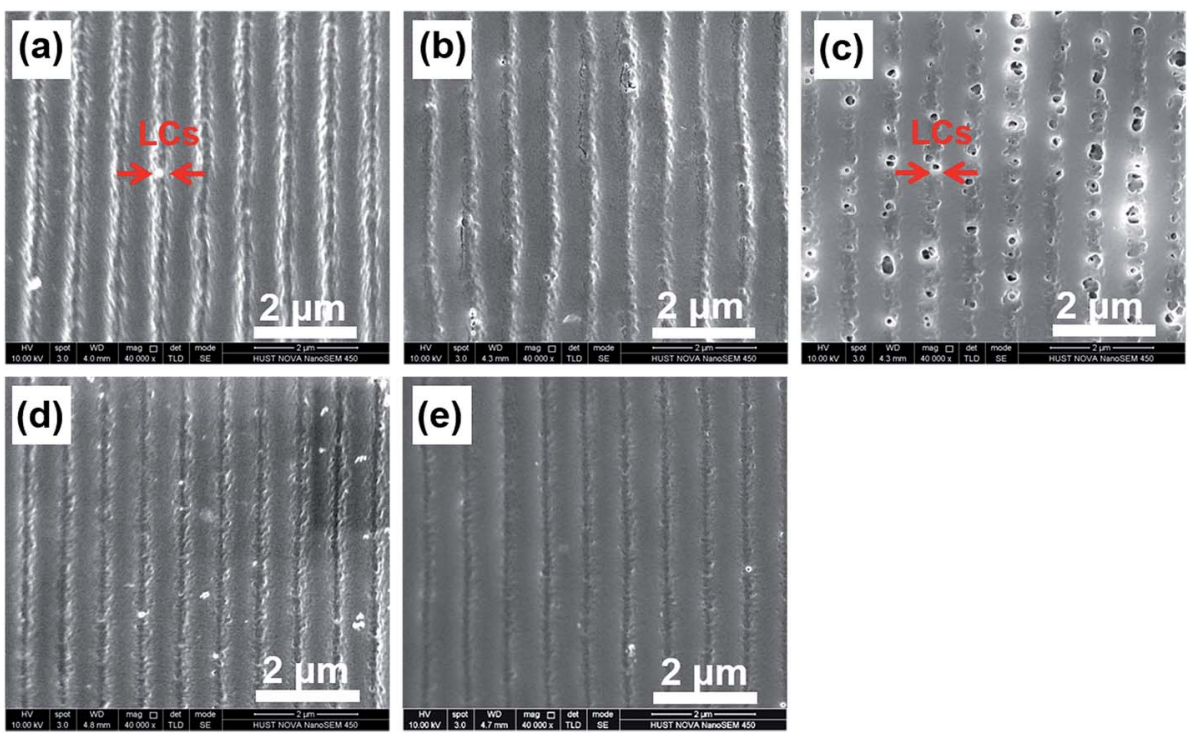

Fig. 4 SEM images of HPDLC gratings with varied content of LC 4OCB after removing the 4OCB and P0616A with $n$-hexane. The dark holes with bright edges represent the original locations of the LC droplets. The LC 40 CB content is (a) 0 wt\%, (b) 2 wt\%, (c) 5 wt\%, (d) 8 wt\% and (e) 11 wt\%, respectively.

increase of $4 \mathrm{OCB}$ concentration from zero to $11 \%$, the nematicisotropic phase transition temperature of the P0616A/4OCB mixtures gradually increases from 58 to $63{ }^{\circ} \mathrm{C}$ (Fig. 2c), indicating that the nucleation capability of the LC mixture increases, which is expected to offer shorter nucleation time during holographic photopolymerization. Considering the HPDLCs with $5 \mathrm{wt} \%$ of $4 \mathrm{OCB}$, the gelation time has been noted to decrease from $63 \pm 6$ to $51 \pm 9 \mathrm{~s}$ compared with the pristine. Under such condition, if the diffusion time of the LCs is smaller than the nucleation time, while the nucleation and polymerization gelation speed is approximately on the same order, discrete LC droplets are expected in the LCs-rich regions (Scheme 2, case A). With the further increase of the 4OCB content to $11 \mathrm{wt} \%$, the nucleation time continues to decrease while the gelation time increases (e.g., from $51 \pm 9$ to $58 \pm 7 \mathrm{~s}$ ), the nucleation is envisioned to occur faster than the polymerization gelation, which in turn results in well-defined phase separation with alternating LC lamellae and polymer lamellae (Scheme 2, case B).

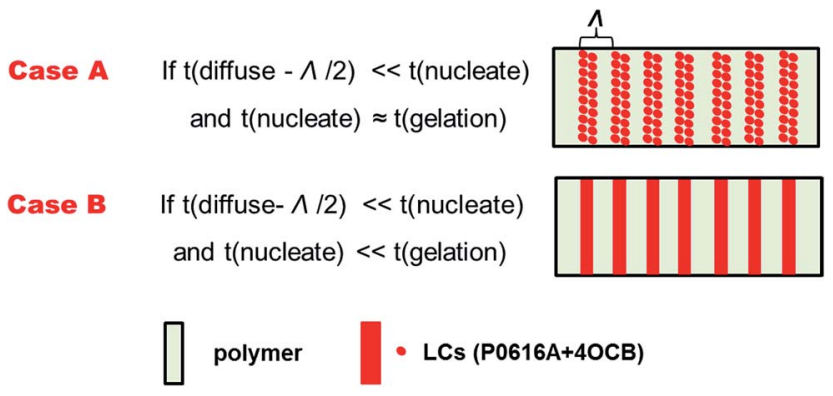

Scheme 2 Schematic representation of the extreme morphologies of HPDLC gratings based on the competition of diffusion, nucleation and gelation.
The morphologies were further investigated using AFM. Fig. 5 shows a three-dimensional view of the transmission gratings with different content of LC 4OCB. The surfaces of the five gratings show sinusoidal type profiles. The grating without 4OCB shows a smooth surface (Fig. 5a). As the LC content increases to 2 and $5 \mathrm{wt} \%$, the AFM images present rough surfaces (Fig. $5 \mathrm{~b}$ and c) that is consistent with the SEM results. Further increasing the 4OCB content to 8 and $11 \mathrm{wt} \%$ results in much smoother grating surfaces again (Fig. $5 \mathrm{~d}$ and e). In addition, an interesting feature observed with AFM is that the depth of the gratings slightly decreases when doped with $5 \mathrm{wt} \%$ of $4 \mathrm{OCB}$. The average depth of the grating with $5 \mathrm{wt} \%$ of $4 \mathrm{OCB}$ is found to be $94 \pm 4 \mathrm{~nm}$; this lower depth is ascribed to the disconnected LC droplets in the LCs-rich phase. The polymer walls in the LCs-rich phase prevent the penetration of the pyramidal AFM tip. Because of the limited resolution of the AFM tip, only rough surface rather than droplet-like structure can be detected.

\subsection{Effect of LC 4OCB content on the diffraction efficiency and light scattering loss of HPDLCs gratings}

The $p$-polarized diffraction efficiency of the HPDLC gratings with varied content of $4 \mathrm{OCB}$ are displayed in Fig. 6a. It can be seen that the diffraction efficiency of the pristine is up to $95 \pm$ $2 \%$. With an increase of $4 \mathrm{OCB}$ content to $2,5,8$ and $11 \mathrm{wt} \%$, the diffraction efficiency of HPDLC gratings does not change significantly, offering values of $94 \pm 2 \%, 92 \pm 3 \%, 93 \pm 3 \%$ and $95 \pm 2 \%$, respectively. Nevertheless, the light scattering loss of HPDLC gratings increases slightly from $10.1 \pm 1.4 \%$ to $13.6 \pm$ $2.7 \%$ as the $4 \mathrm{OCB}$ content increases from zero to $5 \mathrm{wt} \%$ and then decreases back to around $9 \%$ when further increasing the $4 \mathrm{OCB}$ content. 
(a)

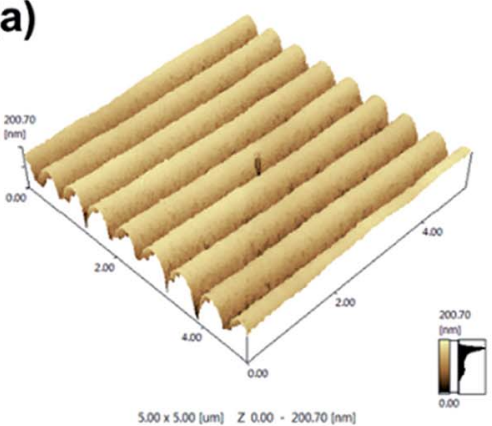

(d)

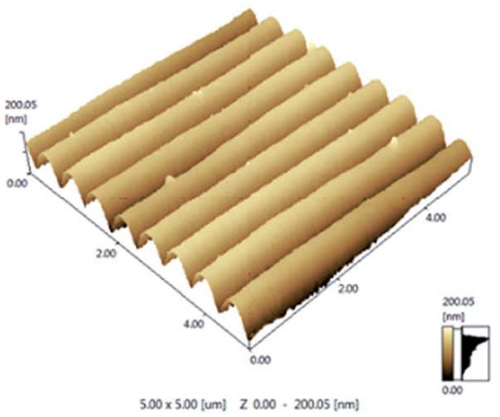

(b)

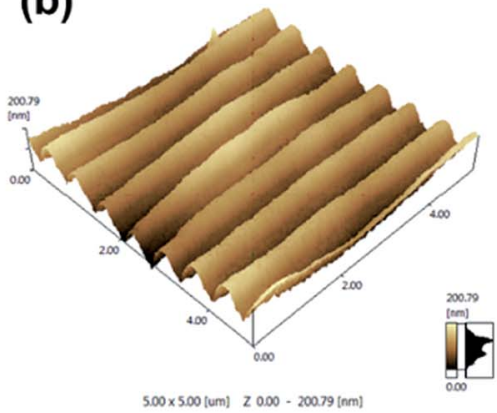

(e)

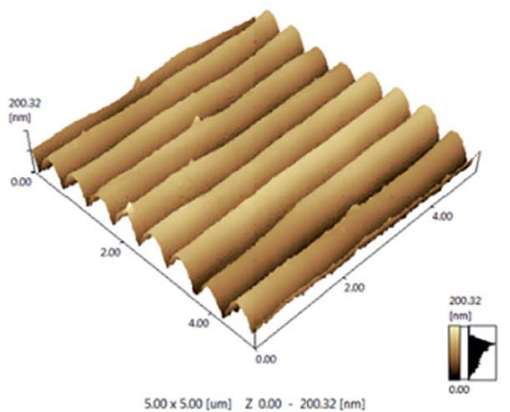

(c)





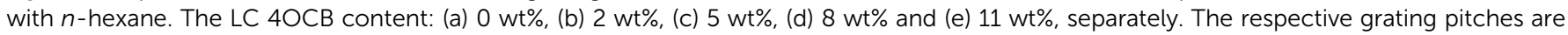

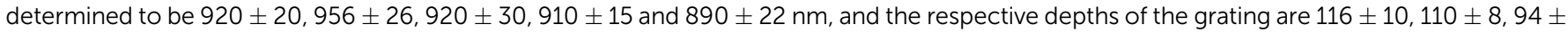
$4,111 \pm 5$ and $102 \pm 7 \mathrm{~nm}$.

In principle, a great mismatch of the refractive index between the polymer and LCs is a basic requirement for HPDLCs with high diffraction efficiency. ${ }^{17}$ The enhanced refractive index mismatch highly relies on the improved phase separation. Because the phase separation increases with the molecular weight of growing polymer, high conversion of monomers into polymer as well as a large solubility parameter gap between the polymer and LCs are of basic concern to obtain good phase separation. ${ }^{48}$ As shown in eqn (3), the diffraction efficiency in HPDLC gratings can be theoretically predicted by Kogelnik coupled-wave theory, ${ }^{23}$

$$
\eta=\sin ^{2}\left[\frac{2 f\left(n_{\mathrm{LC}}-n_{\mathrm{p}}\right) \sin (\beta \pi) L}{\lambda_{\text {probe }} \cos \theta_{\mathrm{B}}}\right]
$$

where $n_{\mathrm{LC}}$ and $n_{\mathrm{p}}$ are respectively the refractive indices of LC and polymer, $f$ represents the volume fraction of LCs in the LCrich region, $\beta$ is defined as the ratio of LC-rich region width to the grating period, $L$ is the grating thickness, $\lambda_{\text {probe }}$ is the wavelength of probe beam, and $\theta_{\mathrm{B}}$ is the grating's Bragg angle. $\lambda_{\text {probe }}, \theta_{\mathrm{B}}$ and $L$ should not be responsible for the difference in the diffraction efficiency. Thus, diffraction efficiency is attributed to the $\left(n_{\mathrm{LC}}-n_{\mathrm{p}}\right), f$ and $\beta$. When the LC 4 OCB content is
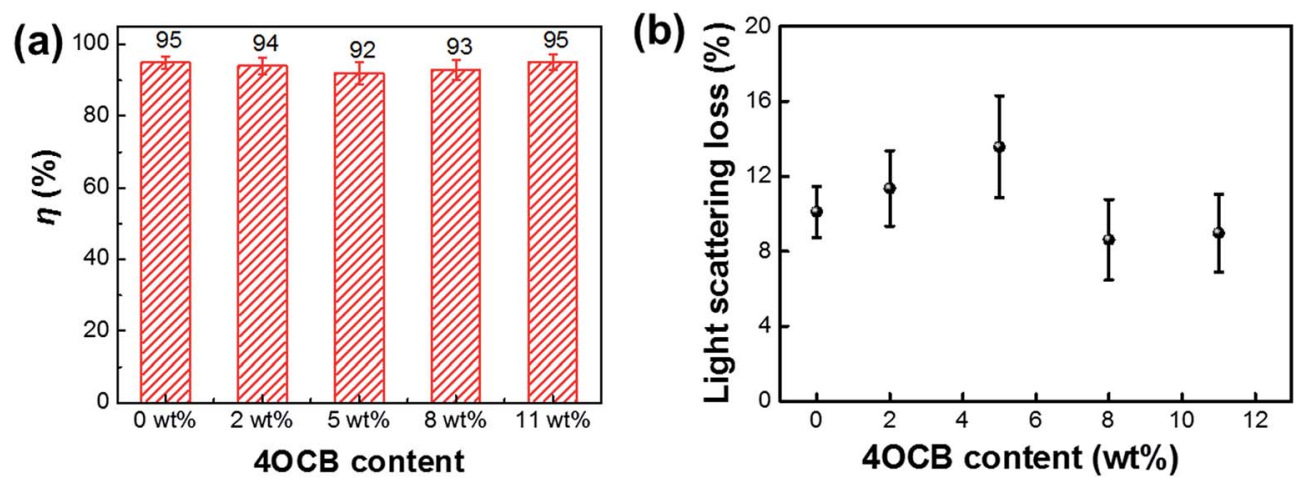

Fig. 6 (a) $p$-Polarized diffraction efficiency, $\eta$, and (b) light scattering loss for the HPDLC gratings with varied content of $4 O C B$. 
$5 \mathrm{wt} \%$, the grating is incapable of forming laminar phase separated structures. Some LC molecules dissolve in the polymer and a small amount of monomers remain in the LCs-rich regions (Fig. 4c), resulting in inferior refractive index mismatch between the polymer and LCs rich phases. Therefore, $\left(n_{\mathrm{LC}}-n_{\mathrm{p}}\right)$ and $f$ decrease slightly although $\beta$ increases from $44 \%$ to $47 \%$, which eventually leads to a slight decrease of diffraction efficiency. In addition, the LC molecules in the HPDLC grating containing $5 \mathrm{wt} \%$ of $4 \mathrm{OCB}$ are subject to extensive coalescence which leads to larger LC droplets. The larger LC droplets cause light scattering, as displayed in Fig. 6b, which also diminishes the diffraction efficiency. Weaker anisotropy of LCs in the HPDLC gratings is also noted for the gratings with larger LC droplets (Fig. S3 in the ESI $\dagger$ ). Consequently, the diffraction efficiency of HPDLCs with $5 \mathrm{wt} \%$ of $4 \mathrm{OCB}$ is slightly lower than other gratings. As the LC 4 OCB content is further increased to 8 and $11 \mathrm{wt} \%$, LCs tend to coalesce to form LC stripes with small LC domains, eventually affording scaffolding morphologies (Fig. 4d and e). The well-defined scaffolding morphology are expected to be as the result of enhanced phase separation, which thus results in enhanced diffraction efficiency.

\subsection{Effect of LC 4OCB content on the electro-optical behaviors of HPDLC gratings}

To further understand the fundamental structure-property relation of HPDLCs, we analyzed the normalized diffraction efficiency versus applied voltage. A square wave voltage up to $250 \mathrm{~V}$ with a frequency of $1 \mathrm{kHz}$ is optimized for the measurement. Fig. 7a shows the dependence of the normalized diffraction efficiency of HPDLC gratings on the applied voltage with varied content of $4 \mathrm{OCB}$. The LC molecules with positive dielectric constants orient along the electric field direction when a voltage is applied across the grating, which results in a decrease of the refractive index mismatch and thus the diminished diffraction efficiency. ${ }^{49}$ The voltages at which the diffraction efficiency decreases to $90 \%$ and $10 \%$ of the initial value are considered as the threshold and saturated voltages, respectively. ${ }^{19,50,51}$ When the content of 4OCB increases from zero to $5 \mathrm{wt} \%$, the threshold $\left(V_{\text {th }}\right)$ and saturated $\left(V_{\text {sat }}\right)$ voltages significantly decrease by $80.8 \%$ (i.e., from $12.0 \pm 0.8$ to $2.3 \pm$



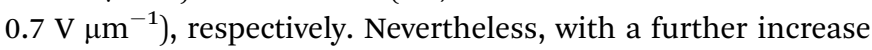

of the $4 \mathrm{OCB}$ content to $11 \mathrm{wt} \%$, these values increase to $11.0 \pm$

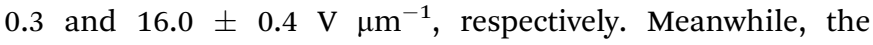
switching-off time increases from $84.7 \pm 13.3$ to $102.5 \pm 13.8 \mathrm{~ms}$ when increasing the $4 \mathrm{OCB}$ content to $5 \mathrm{wt} \%$ and then deceases to $35.7 \pm 11.0 \mathrm{~ms}$ when further increasing the 4OCB content to $11 \mathrm{wt} \%$ (Fig. 7b), primarily because of varied anchoring energy at the polymer/LC interface. Larger LC droplets usually suffer from smaller anchoring energy because of the decreased specific surface area, and thus give rise to longer switching-off time. Detailed data are summarized in Table 3.

The critical electric field for driving HPDLCs can be written as follows, ${ }^{23}$

$$
E_{\mathrm{c}}=\frac{1}{3 b}\left(\frac{\sigma_{\mathrm{LC}}}{\sigma_{\mathrm{p}}}+2\right)\left[\frac{k_{33}\left(l^{2}-1\right)}{\Delta \varepsilon}\right]^{1 / 2}
$$

where $b, \sigma_{\mathrm{LC}}$ and $\sigma_{\mathrm{p}}$ are the length of the semi-major axis of the LC droplet, conductivity of LCs-rich and polymer-rich phases, separately. $k_{33}, \Delta \varepsilon$ and $l$ are the bending constant, dielectric anisotropy and shape anisotropy of neat LCs, respectively. As depicted by eqn (4), the driving voltage difference mainly relies on the size and shape anisotropy of LCs-rich domains. The HPDLCs grating containing $5 \mathrm{wt} \%$ of $4 \mathrm{OCB}$ gives the largest LCs droplets with an average size of $70 \pm 20 \mathrm{~nm}$ and small shape anisotropy, thus offering the lowest driving voltage. In addition, the electro-optical performance of HPDLC gratings is dependent upon the interface anchoring strength between the LC droplets and polymer host. ${ }^{31,50}$ The twisting energy when an electric field is applied to re-orientate of the director of LC molecules inside LC droplets is generally lower than the anchoring energy on LCs by the polymer matrix. Thus, the LCs are able to return to their original status after switching off the electric field. A low interface anchoring energy favors the orientation of LC director along the applied field at a substantially low voltage. However, lower interface anchoring energy gives rise to increased switch-off time, as depicted in Fig. 7 and Table 3 . The interface anchoring energy, $W_{\mathrm{B}}$, between the LC droplets and polymer can be expressed according to Berreman's groove model, ${ }^{52}$

$$
W_{\mathrm{B}}=0.25 K A^{2}\left(\frac{2 \pi}{\Lambda}\right)^{3}
$$
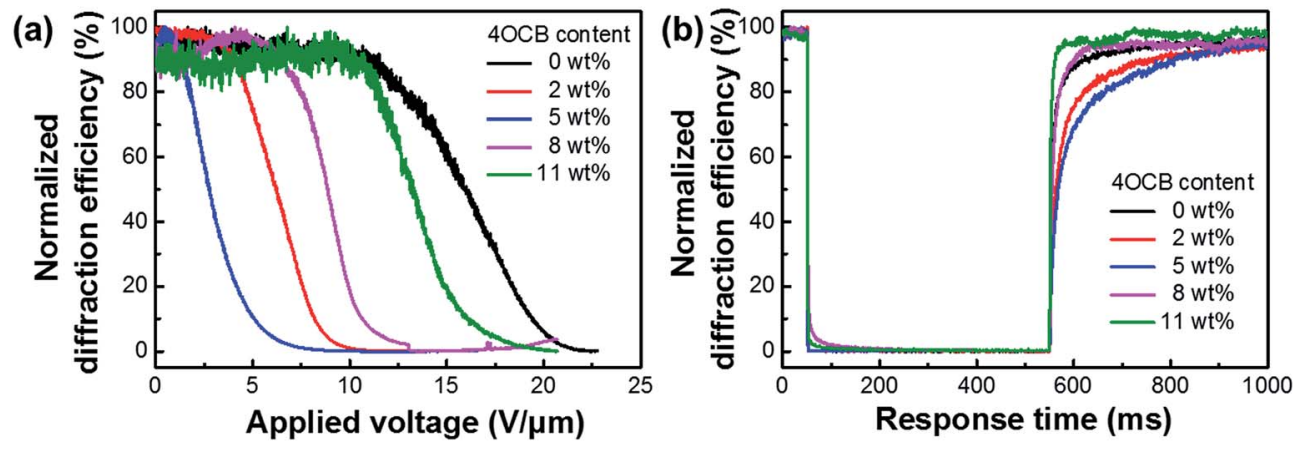

Fig. 7 Normalized diffraction efficiency versus (a) applied voltage and (b) response time for the HPDLC gratings with varied content of 4 OCB. 
Table 3 The interface anchoring energy between the LCS and polymer, and the electro-optical parameters of the HPDLC gratings with varied content of $4 \mathrm{OCB}$

\begin{tabular}{|c|c|c|c|c|c|}
\hline 4OCB (wt\%) & 0 & 2 & 5 & 8 & 11 \\
\hline$W_{\mathrm{B}}\left(\mu \mathrm{N} \mathrm{m}^{-1}\right)$ & 1.1 & 0.9 & 0.7 & 1.0 & 0.9 \\
\hline$V_{\mathrm{th}}\left(\mathrm{V} \mu \mathrm{m}^{-1}\right)$ & $12.0 \pm 0.8$ & $4.9 \pm 0.7$ & $2.3 \pm 0.9$ & $9.5 \pm 1.1$ & $11.0 \pm 0.3$ \\
\hline$\tau_{\text {off }}(\mathrm{ms})$ & $84.7 \pm 13.3$ & $99.8 \pm 9.2$ & $102.5 \pm 13.8$ & $43.7 \pm 9.3$ & $35.7 \pm 11.0$ \\
\hline CR & 18.0 & 26.0 & 23.0 & 22.0 & 13.0 \\
\hline
\end{tabular}

where $A$ is the depth of the groove formed by the polymer after exposure, $\Lambda$ is the grating pitch, and $K$ is the Frank elastic constant of LCs $\left(\sim 10^{-12} \mathrm{~N}\right)$. It is clear that the interface anchoring energy depends on the groove depth and grating pitch. The relationship between interface anchoring energy and 4OCB concentration is also illustrated in Table 3 . It can be seen that adding $5 \mathrm{wt} \%$ of $4 \mathrm{OCB}$ can effectively reduce the interface anchoring energy and thus further decrease the driving voltage of HPDLC gratings.

In addition to the driving voltage and response time, contrast ratio (CR) is also an indispensable factor in evaluating the electro-optical properties of HPDLC gratings when they are applied as electro-optical devices. Eqn (6) can be used to calculate the CR (Table 3$),{ }^{53}$

$$
\mathrm{CR}=10 \log \left(\frac{\eta_{\max }}{\eta_{\min }}\right)
$$

where $\eta_{\max }$ is the highest diffraction efficiency and $\eta_{\min }$ is the lowest diffraction efficiency. We note that a proper loading of 4OCB (2-8 wt\%) gives higher CR values than other samples, which indicates that the low voltage-on state diffraction efficiency and high voltage-off state diffraction efficiency are given. The formed HPDLC gratings are stable under the temperature up to $104{ }^{\circ} \mathrm{C}$ as shown in Fig. S4 in the ESI. $\dagger$ Theoretically, HPDLC devices can be used for several years at room temperature if not destroyed by a high electric field.

\section{Conclusions}

In summary, we have synthesized the non-room-temperature LC $4 \mathrm{OCB}$ and experimentally investigated the effects of $40 \mathrm{OB}$ on the morphology and electro-optical properties of HPDLC transmission gratings. Due to the good compatibility between the 4OCB and P0616A, the 4OCB participated in the phase separation process as a radical inert part and jointly formed the LCs-rich phase. The introduction of $40 \mathrm{OB}$ was found to facilitate the formation of larger LC droplets in the LCs-rich regions at a proper loading. The morphology and performance variations come from the competition among LC diffusion, LC nucleation and system gelation. The $40 C B$ is believed to offer faster nucleation during the process of holographic photopolymerization induced phase separation. A decrease in the interface anchoring energy was also noticed between the LCs and polymer when incorporating 4OCB. After doping $5 \mathrm{wt} \%$ of 4OCB into the HPDLCs, the threshold and saturated voltages significantly decreased by $80.8 \%$ (i.e., from $12.0 \pm 0.8$ to $2.3 \pm$
$0.9 \mathrm{~V} \mu \mathrm{m}^{-1}$ ) and $73.2 \%$ (i.e., from $19.0 \pm 0.6$ to $5.1 \pm$ $0.7 \mathrm{~V} \mathrm{\mu m}^{-1}$ ), respectively, in comparison with the pristine, yet offering high diffraction efficiency of $92 \pm 3 \%$. This simple LC mixing protocol paves the way to fabricate diffractive optical elements with both high diffraction efficiency and low driving voltage.

\section{Conflicts of interest}

The authors declare that there is no conflict of interest.

\section{Acknowledgements}

We thank the financial support from the National Natural Science Foundation of China (51503045, 51433002, 51773073). The Natural Science Foundation of Hubei Scientific Committee (2016CFA001) and the Fundamental Research Funds for the Central Universities (2017KFYXJJ165) are also grateful.

\section{References}

1 C. Y. Li and T. J. Bunning, Curr. Opin. Chem. Eng., 2013, 2, 63. 2 R. L. Sutherland, L. V. Natarajan, V. P. Tondiglia and T. J. Bunning, Chem. Mater., 1993, 5, 1533.

3 T. J. Bunning, L. V. Natarajan, V. P. Tondiglia and R. L. Sutherland, Annu. Rev. Mater. Sci., 2000, 30, 83.

4 K. N. Wang, J. H. Zheng, Y. R. Liu, H. Gao and S. L. Zhuang, Opt. Commun., 2017, 392, 128.

5 Y. J. Zhao, Z. Y. Xie, H. C. Gu, C. Zhu and Z. Z. Gu, Chem. Soc. Rev., 2012, 41, 3297.

6 V. P. Tondiglia, L. V. Natarajan, R. L. Sutherland, D. Tomlin and T. J. Bunning, Adv. Mater., 2002, 14, 187.

7 K. N. Wang, J. H. Zheng, H. Gao, F. Y. Lu, L. J. Sun, S. Yin and S. L. Zhuang, Opt. Express, 2015, 23, 31436.

8 L. J. Liu, L. Xuan, G. Y. Zhang, M. H. Liu, L. F. Hu, Y. G. Liu and J. Ma, J. Mater. Chem. C, 2015, 3, 5566.

9 W. B. Huang, Z. H. Diao, Y. G. Liu, Z. H. Peng, C. L. Yang, J. Ma and L. Xuan, Org. Electron., 2012, 13, 2307.

10 M. H. Liu, Y. G. Liu, Z. H. Peng, S. X. Wang, Q. D. Wang, Q. Q. Mu, Z. L. Cao and L. Xuan, Materials, 2017, 10, 509.

11 M. H. Liu, Y. G. Liu, G. Y. Zhang, Z. H. Peng, D. Y. Li, J. Ma and L. Xuan, J. Phys. D: Appl. Phys., 2016, 49, 465102.

12 H. Y. Peng, G. N. Chen, M. L. Ni, Y. Yan, J. Q. Zhuang, V. A. L. Roy, R. K. Y. Li and X. L. Xie, Polym. Chem., 2015, 6, 8259. 
13 H. Y. Peng, S. G. Bi, M. L. Ni, X. L. Xie, Y. G. Liao, X. P. Zhou, Z. G. Xue, J. T. Zhu, Y. Wei, C. N. Bowman and Y.-W. Mai, J. Am. Chem. Soc., 2014, 136, 8855.

14 G. N. Chen, M. L. Ni, H. Y. Peng, F. H. Huang, Y. G. Liao, M. K. Wang, J. T. Zhu, V. A. L. Roy and X. L. Xie, ACS Appl. Mater. Interfaces, 2017, 9, 1810.

15 A. Ogiwara, M. Watanabe and Y. Ito, Appl. Opt., 2017, 56, 4854.

16 M. L. Ni, G. N. Chen, H. W. Sun, H. Y. Peng, Z. F. Yang, Y. G. Liao, Y. S. Ye, Y. K. Yang and X. L. Xie, Mater. Chem. Front., 2017, 1, 294.

17 Y. J. Liu, B. Zhang, Y. Jia and K. S. Xu, Opt. Commun., 2003, 218, 27.

18 M. S. Park and B. K. Kim, Nanotechnology, 2006, 17, 2012.

19 M. De Sarkar, N. L. Gill, J. B. Whitehead and G. P. Crawford, Macromolecules, 2003, 36, 630.

20 R. A. Ramsey and S. C. Sharma, ChemPhysChem, 2009, 10, 564.

21 N. H. Nataj, E. Mohajerani, H. Jashnsaz and A. Jannesari, Appl. Opt., 2012, 51, 697.

22 J. H. Park and B. K. Kim, J. Polym. Sci., Part A: Polym. Chem., 2013, 51, 1255.

23 H. Y. Peng, M. L. Ni, S. G. Bi, Y. G. Liao and X. L. Xie, $R S C$ Adv., 2014, 4, 4420.

24 Y. Fukuda and Y. Tomita, Materials, 2016, 9, 16.

25 K. R. Moon, S. Y. Bae and B. K. Kim, Opt. Mater., 2015, 42, 160.

26 L. V. Natarajan, D. P. Brown, J. M. Wofford, V. P. Tondiglia, R. L. Sutherland, P. F. Lloyd and T. J. Bunning, Polymer, 2006, 47, 4411.

27 J. Y. Woo and B. K. Kim, Liq. Cryst., 2008, 35, 987.

28 J. Y. Woo, E. H. Kim and B. K. Kim, ChemPhysChem, 2008, 9, 141.

29 E. H. Kim, J. Y. Woo, Y. H. Cho and B. K. Kim, Bull. Chem. Soc. Jpn., 2008, 81, 773.

30 J. Y. Woo and B. K. Kim, ChemPhysChem, 2007, 8, 175.

31 J. Klosterman, L. V. Natarajan, V. P. Tondiglia, R. L. Sutherland, T. J. White, C. A. Guymon and T. J. Bunning, Polymer, 2004, 45, 7213.

32 Y. J. Liu, X. W. Sun, H. T. Dai, J. H. Liu and K. S. Xu, Opt. Mater., 2005, 27, 1451.
33 E. H. Kim, J. Y. Woo and B. K. Kim, Macromol. Rapid Commun., 2006, 27, 553.

34 J. D. Busbee, A. T. Yuhl, L. V. Natarajan, V. P. Tongdilia, T. J. Bunning, R. A. Vaia and P. V. Braun, Adv. Mater., 2009, 21, 3659.

35 K. Wang, J. Zheng, K. Gui, D. Li and S. Zhuang, Plasmonics, 2015, 10, 383.

36 K. Wang, J. Zheng, Y. Chen, Q. Wang, K. Gui and S. Zhuang, IEEE Photonics Technol. Lett., 2015, 27, 1048.

37 S. K. Shriyan and A. K. Fontecchio, Opt. Express, 2010, 18, 24842.

38 S. K. Shriyan and A. K. Fontecchio, Proc. SPIE, 2009, 7414, 741407.

39 M. W. Jang and B. K. Kim, J. Mater. Chem., 2011, 21, 19226.

40 B. K. Kim, M. W. Jang, H. C. Park, H. M. Jeong and E. Y. Kim, J. Polym. Sci., Part A: Polym. Chem., 2012, 50, 1418.

41 L. Q. Fei, P. Wang, W. Q. Yang and X. Q. Guo, Appl. Chem. Ind., 2013, 42, 803.

42 T. F. Scott, B. A. Kowalski, A. C. Sullivan, C. N. Bowman and R. R. McLeod, Science, 2009, 324, 913.

43 H. H. Winter and F. Chambon, J. Rheol., 1986, 30, 367.

44 T. Tsuji, K. Kubo, A. Mori and Y. Nishimura, Talanta, 2001, $55,485$.

45 Y. Wang, H. G. Yoon, H. K. Bisoyi, S. Kumar and Q. Li, J. Mater. Chem., 2012, 22, 20363.

46 Y. Bai, X. Chen, X. Wan, Q. F. Zhou, H. Liu, B. Zhang and Q. Gong, Appl. Phys. B: Lasers Opt., 2001, 73, 35.

47 T. J. Bunning, L. V. Natarajan, V. P. Tondiglia, R. L. Sutherland, R. Haaga and W. W. Adams, Proc. SPIE, 1996, 2651, 44.

48 J. A. Jung and B. K. Kim, Opt. Commun., 2005, 247, 125.

49 A. F. Senyurt, G. Warren, J. B. Whitehead and C. E. Hoyle, Polymer, 2006, 47, 2741.

50 M. De Sarkar, J. Qi and G. P. Crawford, Polymer, 2002, 43, 7335.

51 M. D. Schulte, S. J. Clarson, L. V. Natarajan, D. W. Tomlin and T. J. Bunning, Liq. Cryst., 2000, 27, 467.

52 Z. Zheng, F. Guo, Y. Liu and L. Xuan, Appl. Phys. B: Lasers Opt., 2008, 91, 17.

53 Y. J. Liu, X. W. Sun, J. H. Liu, H. T. Dai and K. S. Xu, Appl. Phys. Lett., 2005, 86, 041115. 